\title{
Mesenchymal stem cells play a potential role in regulating the establishment and maintenance of epithelial-mesenchymal transition in MCF7 human breast cancer cells by paracrine and induced autocrine TGF- $\beta$
}

\author{
QILIN XU ${ }^{1 *}$, LIANG WANG ${ }^{1,2^{*}}$, HONGLING LI $^{1}$, QIN HAN $^{1}$, JING LI $^{1}$, \\ XUEBIN ${ }^{1}{ }^{1}$, SHAN HUANG ${ }^{1}$ and ROBERT CHUNHUA ZHAO ${ }^{1}$ \\ ${ }^{1}$ Institute of Basic Medical Sciences, Chinese Academy of Medical Sciences and Peking Union Medical College, \\ Beijing 100005; ${ }^{2}$ Shengli Oilfield Center Hospital, Dongying 257000, Shandong, P.R. China
}

Received March 22, 2012; Accepted May 11, 2012

DOI: 10.3892/ijo.2012.1541

\begin{abstract}
Although the epithelial-mesenchymal transition (EMT) is a normal process that occurs during development, it is thought to be associated with cancer progression and metastasis. Emerging evidence links mesenchymal stem cells (MSCs) in the tumor microenvironment with the occurrence of EMT in cancer progression. In this study, the human breast cancer cell line MCF7 was co-cultured with human adiposederived MSCs (hAD-MSCs) in a transwell system. Co-cultured cells were analyzed for changes in cellular morphology, EMT markers, protein expression and tumor characteristics. We found that co-cultured MCF7 cells underwent EMT and established a stable mesenchymal phenotype after prolonged co-culturing. Here, we demonstrate that paracrine transforming growth factor- $\beta 1$ (TGF- $\beta 1$ ) secreted by hAD-MSCs regulated the establishment of EMT in MCF7 cells by targeting the ZEB/miR-200 regulatory loop. The downregulation of paracrine TGF- $\beta 1$ levels can inhibit and reverse the EMT progress by downregulating ZEB1/2 and upregulating miR-200b and miR-200c. The maintenance of a stable mesenchymal state by
\end{abstract}

Correspondence to: Dr Robert Chunhua Zhao, Institute of Basic Medical Sciences, Chinese Academy of Medical Sciences and Peking Union Medical College, 5 Dongdansantiao, Beijing 100005, P.R. China

E-mail: chunhuaz@public.tpt.tj.cn

*Contributed equally

Abbreviations: EMT, epithelial-mesenchymal transition; MSCs, mesenchymal stem cells; hAD-MSCs, human adipose-derived MSCs; MCF7-Co, MCF7 cells co-cultured with hAD-MSCs; MCF7-M, MCF7 cells with stable mesenchymal state; ZO-1, zonula occludens 1

Key words: mesenchymal stem cells, epithelial-mesenchymal transition, paracrine TGF- $\beta$, autocrine TGF- $\beta$, ZEB/miR-200 regulatory loop, metastasis
MCF7 cells required the establishment of autocrine TGF- $\beta$ signaling to drive and sustain ZEB expression, which had been initiated by the prolonged co-culturing with hAD-MSCs. These results suggest that MSCs may promote breast cancer metastasis by stimulating and facilitating the EMT process.

\section{Introduction}

Breast cancer, which starts as a local disease and can metastasize to distant organs, is the second leading cause of cancer-related mortality in women $(1,2)$. The conversion of early stage tumors into invasive malignancies has been associated with the activation of the epithelial-mesenchymal transition (EMT), defined as changes in cell phenotype from an epithelial to a mesenchymal state, which is both a fundamental event and a hallmark in tumorigenesis (3-9). The acquisition of mesenchymal properties through EMT can promote the detachment of cancer cells from the primary tumor and facilitate their subsequent migration, thus allowing the progression of metastasis to proceed $(8,10)$. Recent studies have shown that process is also associated with the acquisition of self-renewing, tumor-initiating properties (11-13). Despite the now recognized role of EMT in the metastatic cascade, how stimuli-induced EMT occurs at the primary tumor site remains largely unknown.

Epithelial cell tumors develop in a symbiotic manner with the surrounding stroma. Tumor cells actively recruit cells, including MSCs, into the tumor microenvironment, and these cells may subsequently play an important role in facilitating cancer progression (14). MSCs have been shown to affect the morphology and proliferation of cells within their vicinity through cell-to-cell interactions as well as through the secretion of chemotactic cytokines and paracrine factors (15-18). Accumulating evidence suggests that the interactions between MSCs and breast cancer cells may impact the phenotype of the cancer cells and promote their metastatic potential (19-22). A pivotal study by Karnoub et al reported that MSCs enhance breast cancer cell motility, invasion and metastatic potential in vivo through CCL5 signaling, which confirms 
that these paracrine interactions play an important role in the MSC-mediated metastatic spread (20). Further understanding of the interactions between MSCs and breast cancer cells is required to determine the role of MSCs in breast cancer progression or therapeutics.

Among several growth factors that can act as inducers of EMT, TGF- $\beta$ has been found to play an important role at particular stages of development and in disease processes such as fibrosis and cancer metastasis (23-25). TGF- $\beta$ induces EMT activators: a group of transcription factors, including ZEB1 and ZEB2, which repress epithelial gene expression $(26,27)$. Furthermore, ZEB1 and ZEB2 are crucial targets of miR-200 family members (28-30), and all miR-200 members are transcriptional targets of ZEB1 and ZEB2 (31). Thus, ZEB factors and miR-200 family members not only have opposite functions but also reciprocally control the expression of each other. This double-negative feedback loop between miR-200 family members and ZEB1 allows for the plasticity that exists between the cell's epithelial and mesenchymal states $(31,32)$. Therefore, tilting to one side of the feedback loop instead of the other, which allows for the stabilization of either an epithelial or mesenchymal phenotype, may depend on several factors such as the extracellular signals.

In this study, we co-cultured MCF7 human breast cancer cells and hAD-MSCs in a transwell system to observe the effect of hAD-MSCs on MCF7 cells. We aimed to elucidate the role of hAD-MSCs paracrine signaling in the establishment and maintenance of EMT in breast cancer cells and to detect the underlying mechanisms.

\section{Materials and methods}

Culture of human adipose-derived MSCs. Human adipose tissue was obtained from patients undergoing tumescent liposuction following procedures approved by the Ethics Committee at the Chinese Academy of Medical Sciences and Peking Union Medical College. Isolation and cell culturing protocols reported by Cao et al were used in this study (33). hAD-MSCs at passage three were employed.

Immunophenotype analysis. Prior to using them in experiments, MSCs were evaluated for the expression of CD29, CD44, CD105, CD106, CD31, CD34 and HLA-DR. The cells were detached and washed with phosphate-buffered saline (PBS), then incubated with primary antibodies for $30 \mathrm{~min}$ at $4^{\circ} \mathrm{C}$. To detect intracellular antigens, the cells were fixed in $2 \%$ paraformaldehyde at $4^{\circ} \mathrm{C}$ for $15 \mathrm{~min}$ and then permeabilized with $0.1 \%$ saponin at room temperature for $1 \mathrm{~h}$. Working concentrations for primary antibodies (BD Biosciences, USA) were 10-20 ng/ml. Same-species and isotype irrelevant antibodies were used as negative control. After washing with PBS, the cells were incubated with fluorescein isothiocyanate and phycoerythrin-conjugated secondary antibodies at $4^{\circ} \mathrm{C}$ for $30 \mathrm{~min}$. The cells were then resuspended in PBS and analysed by a flow cytometer (FACSCalibur; Becton-Dickinson, San Jose, CA). The results were analysed by CellQuest Pro software (BD Biosciences).

Culture of human tumor cell line. The human breast cancer cell line MCF7 was obtained from the American Type Culture
Collection (Manassas, VA, USA) and grown in H-DMEM (Dulbecco's modified Eagle's medium; Gibco) supplemented with $10 \%$ fetal bovine serum (FBS), $100 \mathrm{U} / \mathrm{ml}$ of penicillin and $100 \mu \mathrm{g} / \mathrm{ml}$ of streptomycin.

Transwell co-culture experiment. In this assay, a transwell system with a $0.3-\mu \mathrm{m}$ pore size permeable membrane (Corning Costar) was used to separate MCF7 cells physically from hAD-MSCs. MCF7 cells were seeded into the upper insert of a transwell system at a density of $1 \times 10^{5}$ cells/well in 6-well plates in MSC culture medium. hAD-MSCs were cultured in the lower chamber of this co-culture system. The transwell co-culture system was used in all experiments.

Cell cycle analysis. Briefly, 5.0× $10^{5}$ cells were harvested and fixed in $75 \%$ cold ethanol, washed twice with cold PBS, and then incubated in PBS containing $50 \mu \mathrm{g} / \mathrm{ml}$ propidium iodide (PI) and $20 \mu \mathrm{g} / \mathrm{ml} \mathrm{RNaseA} \mathrm{for} 30 \mathrm{~min}$ at $37^{\circ} \mathrm{C}$. PI and forward light scattering were detected using a flow cytometer (FACSCalibur; Becton-Dickinson). Experiments were performed in triplicate. A percentage of cells in each phase of the cell cycle was analyzed.

Cell proliferation assay. Cells were seeded in 96-well plates at $2.0 \times 10^{3}$ cells/well. 3-(4,-dimethylthiazol-2-yl)-2,5-diphenyltetrazolium bromide (MTT) assay was used to detect viable proliferating cells at various time points. The absorbance values for positive staining cells were measured using a microplate reader (BioTek, USA) at a $570 \mathrm{~nm}$ wavelength to determine the OD.

Soft-agar colony formation assay. The colony formation assay was performed to determine the in vitro tumorigenesis of both MCF7 cells before and after co-culturing with hAD-MSCs. Wells of a 6-well plate (BD Falcon, USA) were pre-coated with $0.6 \%$ agar containing H-DMEM and $10 \%$ FBS. Cells (1.0x $10^{3}$ cells/well) in $1.5 \mathrm{ml}$ of $0.3 \%$ agar containing H-DMEM and $10 \%$ FBS were then added to the base. Cells were fed with fresh top agar every week. Colonies were counted visually after two weeks by staining using a crystal violet staining kit (Beyotime, China).

In vitro migration and invasion assay. For invasion assays, MCF7 cells were cultured in 24-well Matrigel-coated invasion chambers ( $8-\mu \mathrm{m}$ pore, BD Biosciences). The lower chambers were filled with $0.75 \mathrm{ml} \mathrm{H}$-DMEM containing $10 \% \mathrm{FBS}$ as a chemoattractant. A cell suspension of $5.0 \times 10^{4}$ cells in a volume of $0.5 \mathrm{ml} \mathrm{H}$-DMEM was added to the upper chamber. After the cells were incubated for $24 \mathrm{~h}$ at $37^{\circ} \mathrm{C}$ in a humidified incubator with $5 \% \mathrm{CO}_{2}$, the non-invading cells that remained on the upper surface of the membrane were removed by scraping. The invasive cells attached to the lower surface of the membrane insert were fixed with $4 \%$ paraformaldehyde at room temperature for $15 \mathrm{~min}$ and stained with crystal violet staining solution for $30 \mathrm{~min}$. The number of cells in each chamber was then counted under a microscope.

For the transwell migration assays, $1.0 \times 10^{5}$ cells were plated into the upper chamber of the membrane $(8-\mu \mathrm{m}$ pore, BD Biosciences) that had been pre-coated with fibronectin $(100 \mu \mathrm{g} / \mathrm{ml})$ and $2.5 \%$ bovine serum albumin (BSA). Cells 
Table I. The primers used for the Q-PCR experiment.

\begin{tabular}{lll}
\hline Gene & Forward primer & Reverse primer \\
\hline GAPDH & GGTCACCAGGGCTGCTTTTA & GGATCTCGCTCCTGGAAGATG \\
E-cadherin & CCCACCACGTACAAGGGTC & ATGCCATCGTTGTTCACTGGA \\
$\beta$-catenin & GCTACTCAAGCTGATTTGATGGA & GGTAGTGGCACCAGAATGGATT \\
ZO-1 & CAACATACAGTGACGCTTCACA & GACGTTTCCCCACTCTGAAAA \\
Fibronectin & CCCCATTC CAGGACACTTCTG & GCCCACGGTAACAACCTCTT \\
N-cadherin & GAGGAGTCAGTGAAGGAGTCA & GGCAAGTTGATTGGAGGGATG \\
Vimentin & AGAACTTTGCCGTTGAAGCTG & CCAGAGGGAGTGAATCCAGATTA \\
TWIST & CGGACAAGCTGAGCAAGATT & TGGAGGACCTGGTAGAGGAA \\
SNAIL & AATCGGAAGCCTAACTACAGCG & GTCCCAGATGAGCATTGGCA \\
ZEB1 & AGTGATCCAGCCAAATGGAA & TTTTTGGGCGGTGTAGAATC \\
ZEB2 & AACAAGCCAATCCCAGGAG & GTTGGCAATACCGTCATCCT \\
\hline
\end{tabular}

were incubated for the indicated time periods under standard cell culture conditions. Tumor cells remaining on the top-side of the membrane were removed and cells that had migrated to the bottom-side were fixed and stained as described above. Five pre-selected fields per insert were photographed. After the cells had been stained and photographed, cells that had migrated to the bottom-side were washed using 33\% acetic acid, and then the absorbance values for positive staining cells were measured using a microplate reader (BioTek) at a $570 \mathrm{~nm}$ wavelength to determine the OD.

$R N A$-reverse transcription and real-time PCR. Total RNA was extracted using the Trizol protocol (Invitrogen, USA) and the cDNA was synthesized from the mRNA using the PrimeScript II 1st Strand cDNA Synthesis system (Takara, Japan). Relative expression of the genes of interest was assessed by real-time PCR on an ABI StepOnePlus ${ }^{\mathrm{TM}}$ Fast Real-Time PCR System (Applied Biosystems) using SYBR ${ }^{\circledR}$-Green I-PCR reaction mixture (Takara) and specific primers (Table I). The average of three independent analyses for each gene and sample was calculated and normalized to the endogenous reference control gene GAPDH.

Reverse transcription of miRNAs was performed using the miScript Reverse Transcription Kit (Qiagen, China). Expression of mature miRNAs was determined using miRNA-specific quantitative real-time PCR (Qiagen). Real-time PCR data for mRNA and microRNA were expressed relative to GAPDH or U6, respectively. The expression levels were normalized to U6, an internal control, and measured by the comparative $\mathrm{Ct}\left({ }^{\Delta} \mathrm{Ct}\right)$ method. The miRNA-specific quantitative real-time PCR consisted of 40 cycles $\left(95^{\circ} \mathrm{C}\right.$ for $5 \mathrm{sec}$ and $60^{\circ} \mathrm{C}$ for $\left.34 \mathrm{sec}\right)$ after an initial denaturation step $\left(95^{\circ} \mathrm{C}\right.$ for $\left.10 \mathrm{sec}\right)$.

Western blot analysis. The protein levels of TWIST, SNAIL, ZEB1, ZEB2, and phosphorylated-SMAD2 in MCF7 cells cultured alone or co-cultured with hAD-MSCs were analyzed by western blotting. Cells were harvested in RIPA lysis buffer (Beyotime, China). After quantifying by BCA assay, whole cell protein extracts were separated on an SDS-10\% polyacrylamide gel, and the proteins were transferred to a nitrocellulose membrane (Bio-Rad). The membrane was blocked in TBS containing 5\% dried milk powder $(\mathrm{w} / \mathrm{v})$ and $0.1 \%$ Tween-20, and hybridized with antibodies against TWIST (1:500, Abcam), SNAIL (1:500, Santa Cruz), ZEB1 (1:500, Santa Cruz), ZEB2 (1:500, Santa Cruz), phosphorylated SMAD2 (1:500, Santa Cruz) and $\beta$-actin (1:500, Abcam), respectively. The levels of the above-mentioned proteins were normalized to that of $\beta$-actin as a loading control.

Enzyme-linked immunosorbent assay. Concentrations of VEGF, HGF, NGF, FGF, SDF-1 $\alpha$, TGF $\beta 1$, TGF $\beta 2$, and TGF $\beta 3$ were measured by ELISA (Shanghai Senxiong Technology Industry, Shanghai, China), according to the manufacturer's instructions. Experiments were repeated at least three times. Briefly, cells were treated under the appropriate conditions and cell culture supernatants were collected, centrifuged and filtered through a $0.45 \mu \mathrm{m}$ Steriflip Filter Unit (Millipore, USA). The absorbance $(450 \mathrm{~nm})$ for each sample was analyzed by an ELISA reader and interpolated with a standard curve.

Statistical analysis. Each experiment was repeated at least three times. Statistical significance between treatment and control groups was analyzed using the Student's t-test. The data is represented as the means $\pm \mathrm{SD}$. $\mathrm{P}<0.05$ was regarded as a statistically significant difference.

\section{Results}

Morphology and molecular phenotype of hAD-MSCs. The cell morphology and the growth curve of hAD-MSCs were evaluated after three passages. Cell morphology was characterized by spindle or triangular-shaped adherent cells with good refraction. The cell body contained a round nucleus with uniform refractive index giving the nucleus a clear appearance and one or more prominent nucleoli (Fig. 1A). Immunophenotype analysis showed that hAD-MSCs were $\mathrm{CD}^{2} 9^{+}, \mathrm{CD}_{4} 4^{+}, \mathrm{CD} 105^{+}, \mathrm{CD} 34^{-}, \mathrm{CD} 31^{-}, \mathrm{CD}_{106}{ }^{-}$and HLA-DR (Fig. 1B). These features are consistent with our previous findings (33).

Co-cultured MCF7 cells display EMT changes in a timedependent manner. Fibroblast-like appearances that are 


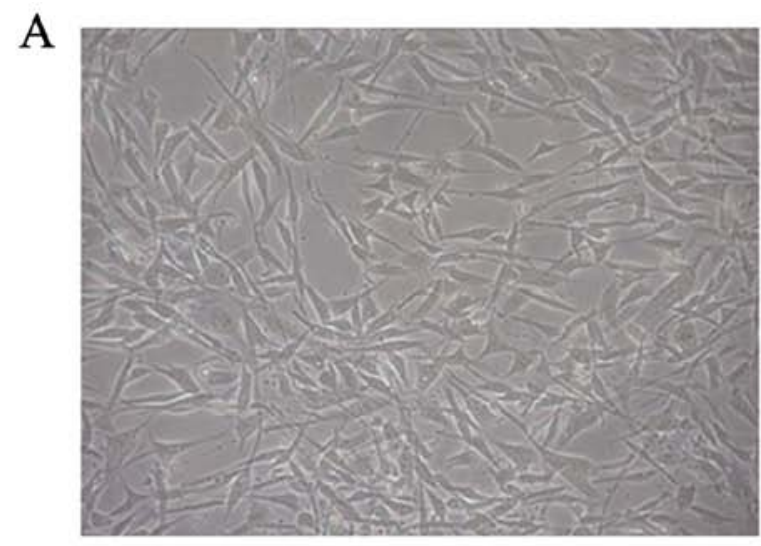

B
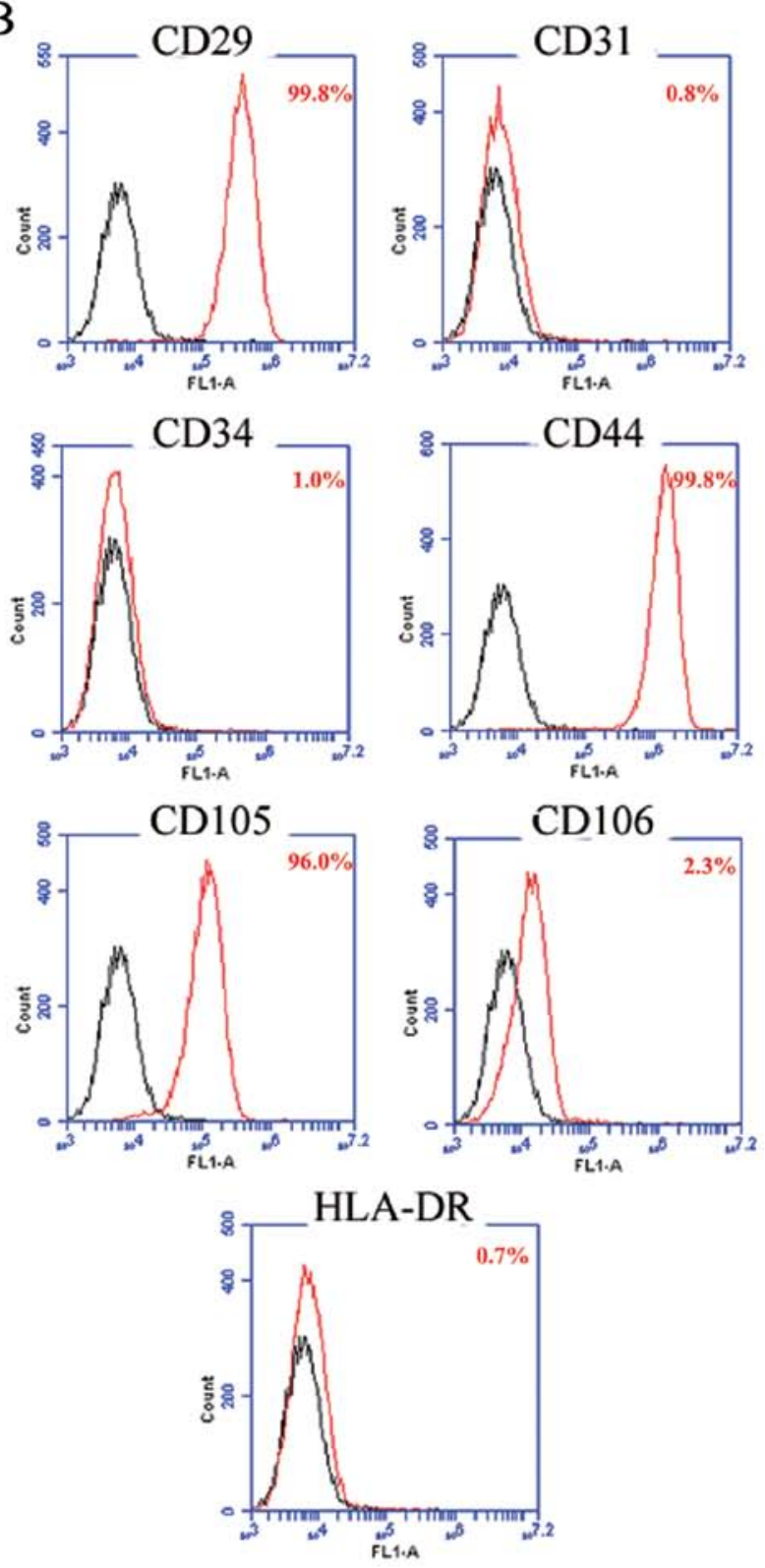

Figure 1. In vitro morphology and immunophenotype of hAD-MSCs. (A) Cell morphology of hAD-MSCs at the 3rd passage (x100). (B) Flow cytometry profile of hAD-MSCs. Red and black-line histograms represent the specific and isotype-matched control antibodies, respectively. consistent with EMT were observed in MCF7 cells after co-culturing with hAD-MSCs for 3 days (MCF7-Co) (Fig. 2A). Quantification of the expression of EMT-related genes on days $0,2,4,6$ and 8 also showed a progressive decrease in epithelial makers (E-cadherin, zonula occludens 1 (ZO-1), $\beta$-catenin) along with a simultaneous increase in mesenchymal markers (N-cadherin, vimentin, fibronectin) (Fig. 2B). To rule out the effect of MSCs culture medium itself on MCF7 cells, MCF7 cells were cultured in MSC culture medium for 8 days, but unlike co-culturing with hAD-MSCs, no EMT-related changes were observed (Fig. 2C).

Then we cultured these mesenchymal MCF7-Co cells obtained at different time points alone in standard MCF7 culture medium, and we observed that the cells obtained at day 8 could maintain a stable mesenchymal phenotype (MCF7-M) and propagate after being passaged five times (Fig. 2D). Quantification by real-time PCR analysis demonstrated the downregulation of epithelial markers and the upregulation of mesenchymal markers in these MCF7-M cells (Fig. 2E).

Effects of hAD-MSCs co-culturing on tumor characteristics of breast cancer cells. To investigate the functional consequences of hAD-MSCs co-culturing on breast cancer cells, we performed flow cytometry, MTT, soft agar colony formation and transwell assays to evaluate the malignant characteristics of both breast cancer cell lines. No significant changes in cell cycle progression, cell proliferative capacity and anchorageindependent growth were observed in MCF7 cells after $72 \mathrm{~h}$ of co-culturing with hAD-MSCs (Fig. 3A-C). In vitro invasion and migration assays showed increased invasive and migratory capacities at 3.8-fold and 2.97-fold respectively of MCF7-Co compared with MCF7 cells (Fig. 3D and E).

TGF- $\beta 1$-stimulated EMT in MCF7 co-cultured with hADMSCs. We hypothesized that the induction of EMT in MCF7 cells co-cultured with hAD-MSCs was due to cytokines secreted by MSCs; thus, we tested the cell culture supernatant using the ELISA assay. The results showed that the level of TGF- $\beta 1$ was significantly higher in the supernatant from co-culture system compared with that from MCF7 cultured alone; differences in the levels of TGF- $\beta 2$ and TGF- $\beta 3$ were very slight (Fig. 4A). Furthermore, the titrated addition of various concentrations of anti-TGF- $\beta 1$ antibody from day 0 to day 6 of the co-culture showed that the expression of phosphorylated SMAD2 (P-SMAD2), which is under the regulation of TGF- $\beta 1$, was reduced in the MCF7 cells in a dose-dependent manner (Fig. 4B). Concurrently, a marked decrease in the level of vimentin transcript and a switch from $\mathrm{N}$-cadherin to E-cadherin was also observed (Fig. 4C). These results indicate that, in our study, the hAD-MSC co-cultureinduced EMT in MCF7 cells was TGF- $\beta 1$-dependent.

TGF- $\beta$ targets the ZEB/miR-200 regulatory loop. To test whether the ZEB/miR-200 regulatory loop was involved in the induction of EMT, we determined the change in ZEB1/2 and miR-200 expression levels in MCF7 cells before and after co-culturing with hAD-MSCs. After $72 \mathrm{~h}$ of co-culturing, the expression of ZEB1 and ZEB2 in MCF7-Co cells was significantly upregulated as observed by both real-time PCR 
A
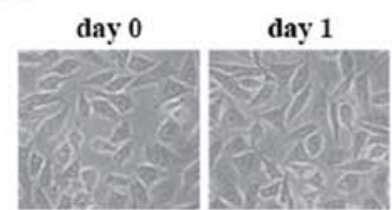

day 3

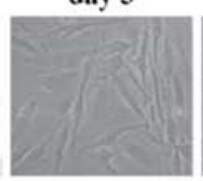

day 5

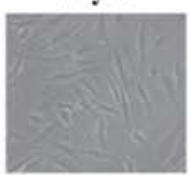

day 8

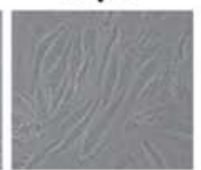

C

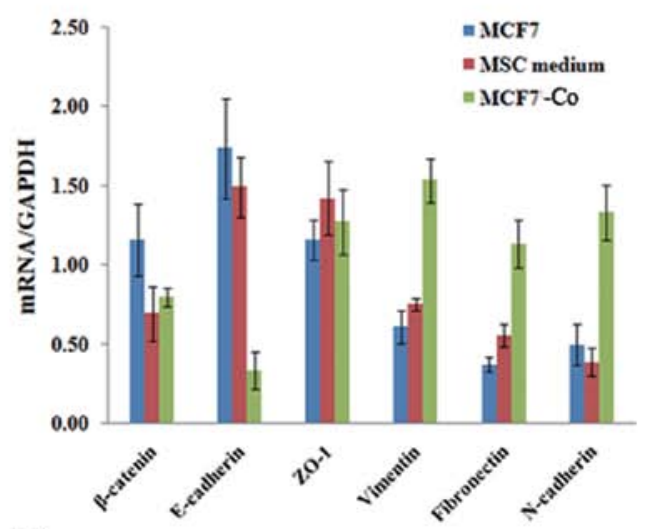

$\mathrm{E}$

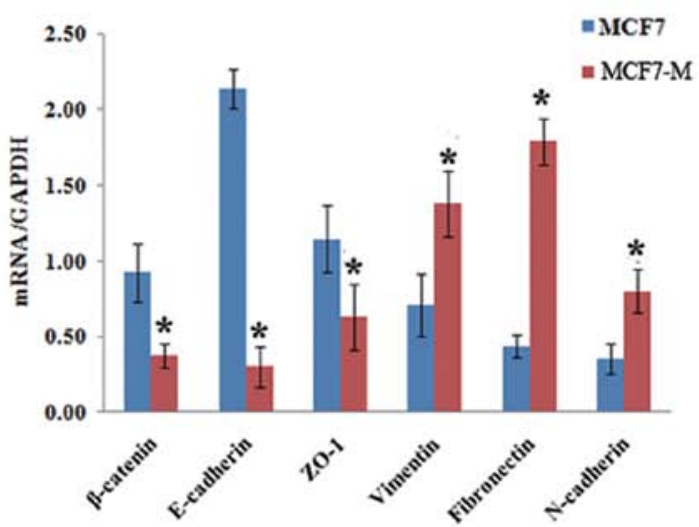

B

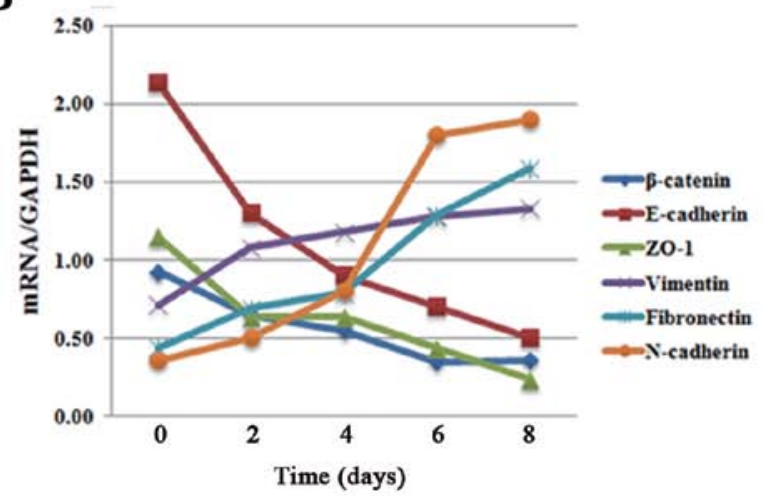

D

Co-cultured with hAD-MSCs

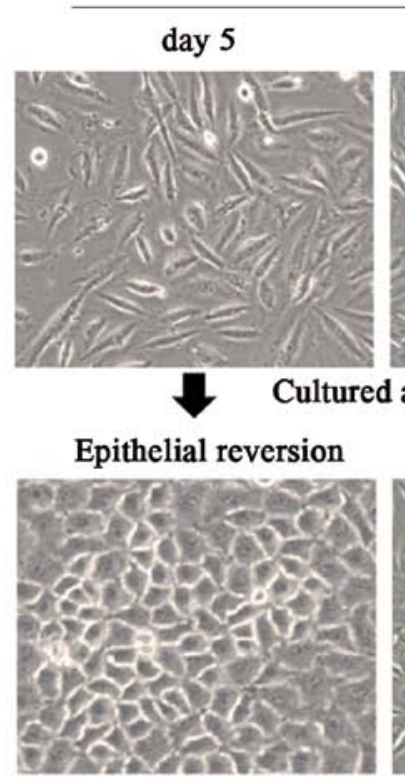

day 8

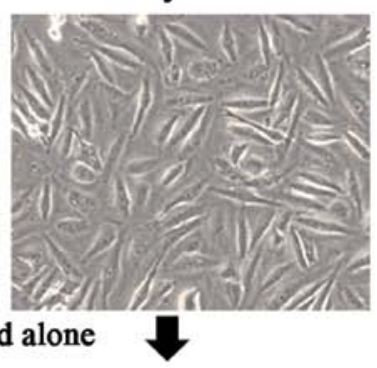

MCF7-M

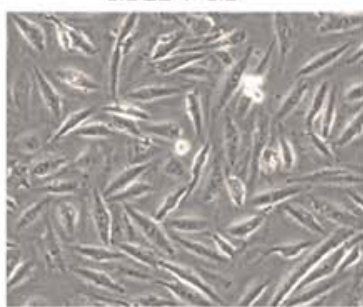

MCF7-M (P5)

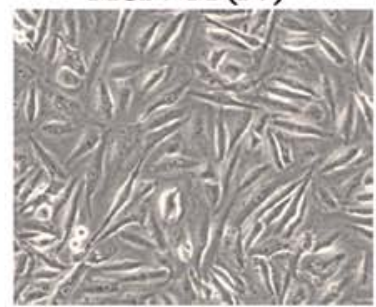

Figure 2. Morphologic changes consistent with EMT in breast cancer cells following co-culture with hAD-MSCs. (A) Morphological changes of MCF7 cells observed at different time points of co-culturing with hAD-MSCs (x200). (B) Trend line of expression of these EMT markers during co-culturing with hAD-MSCs for 8 days. (C) MCF7 cells cultured with only MSC medium did not exhibit significant changes in the expression of these EMT-related markers. MCF7-Co, MCF7 cells co-cultured with hAD-MSCs; MSC medium, cells cultured with MSC culture medium only. * $\mathrm{p}<0.05$. (D) Cell morphology of mesenchymal MCF7-Co cells obtained at day 5 and 8 of co-culturing with hAD-MSCs and followed by culture alone in standard MCF7 culture medium. MCF7-Co cells obtained at day 5 were cultured alone for 7 days resulting in reversion back to an epithelial phenotype (Epithelial reversion). MCF7-Co cells obtained at day 8 of co-culturing maintained a stable mesenchymal phenotype after culturing alone for 12 days (MCF7-M) and also after 5 passages (MCF7-M (P5). (E) Changes of the mRNA levels of various EMT hallmarks in MCF7-M cells. MCF7-M, MCF7 cells with stable mesenchymal state and cultured alone in MCF7 culture medium.

quantification (Fig. 5A) and western blot analysis (Fig. 5B). However, the expression of SNAIL and TWIST did not exhibit significant alterations (Fig. 5A and B). We also observed that miR-200b and miR-200c were downregulated 3-fold and 5-fold in MCF7-Co cells, respectively (Fig. 5C).

Furthermore, the addition of different concentrations of anti-TGF- $\beta 1$ antibody for an over $24 \mathrm{~h}$ period led to a dose- dependent reduction of ZEB1/2 expression in the MCF7-Co cells, which correlated with a decreased expression of phosphorylated SMAD2 (P-SMAD2) (Fig. 4B). Moreover, miR200b and miR-200c transcripts were upregulated (Fig. 3E), and ZEB1 and ZEB2 mRNA levels were downregulated (Fig. 5D). These results are consistent with the idea that the ZEB/miR-200 regulatory loop is regulated by paracrine TGF- $\beta$ signaling. 
A

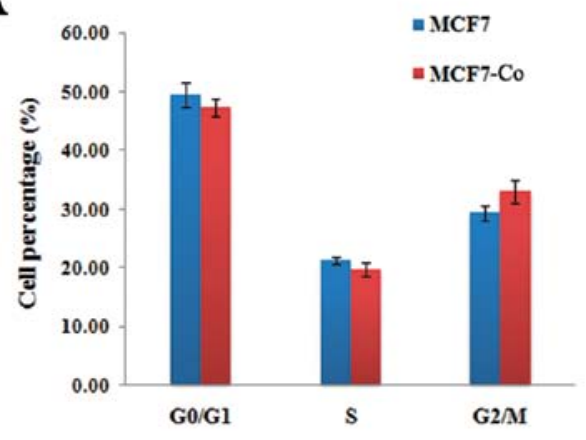

C

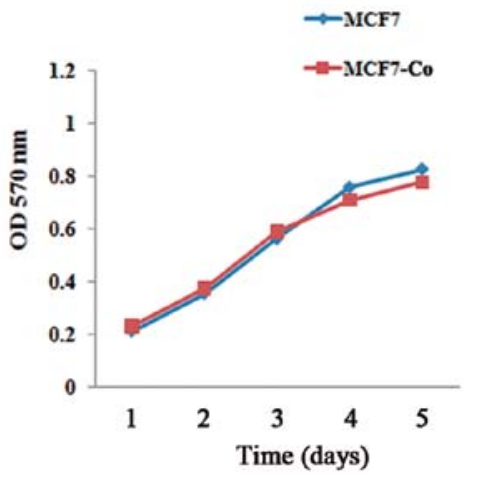

B
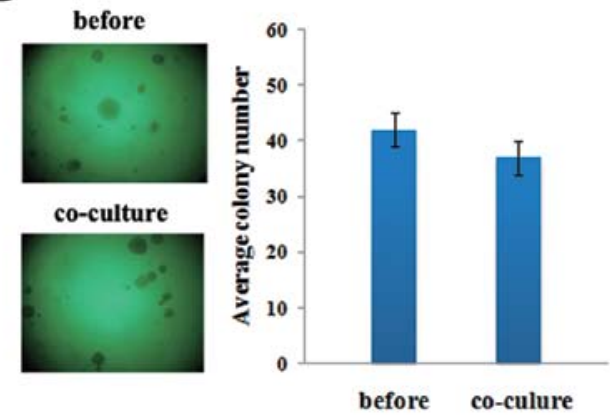

$\mathbf{E}$
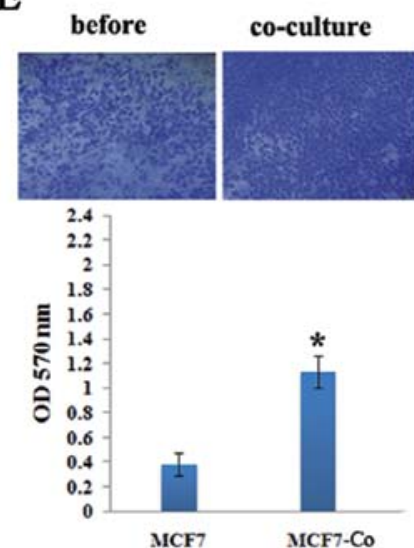

Figure 3. Effect of co-culture with hAD-MSCs on the malignant characteristics of the breast cancer cells. (A) Cell cycle phase determined by flow cytometry assay. (B) Cell proliferation ability determined by MTT assay. (C) Soft agar colony formation assay. (D) Cell invasiveness determined by Matrigel invasion chambers. (E) Cell migration ability assessed by Transwell chamber assay. " $\mathrm{p}<0.05$.

A

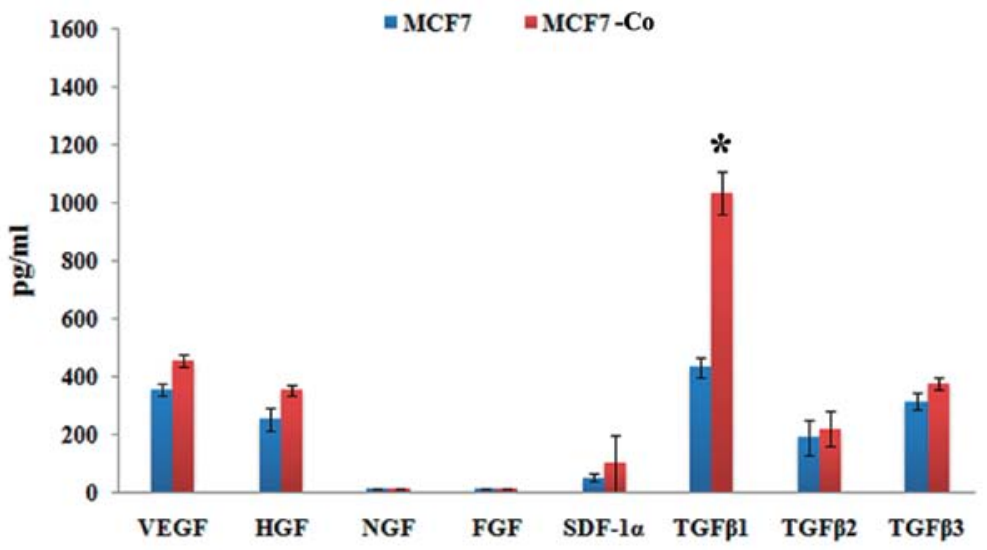

B

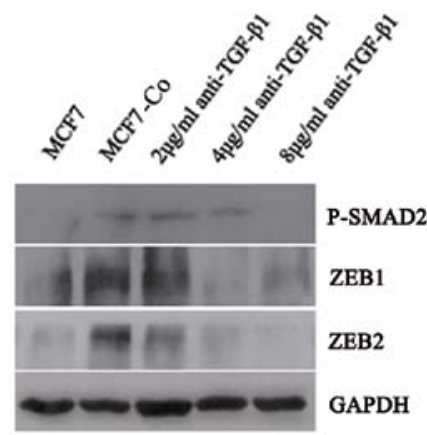

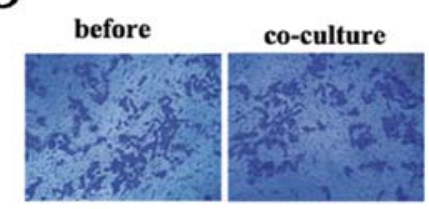

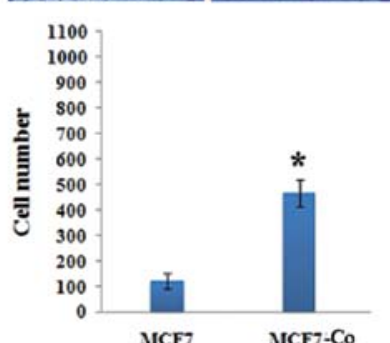

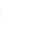

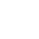


A

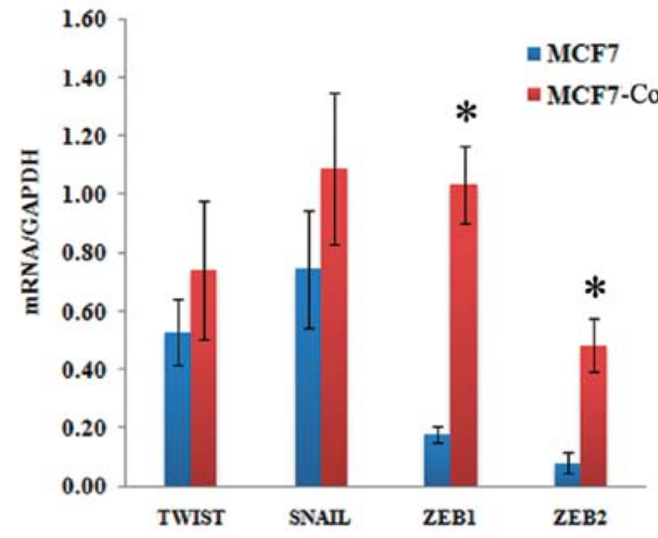

C

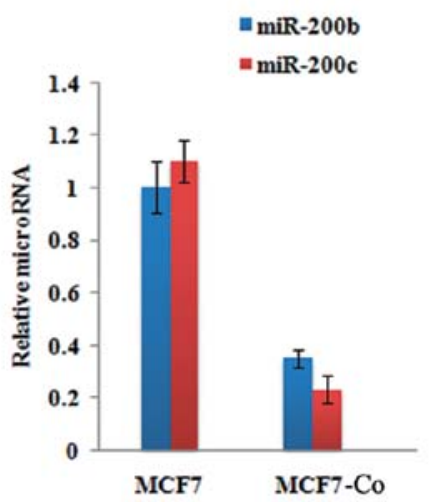

D

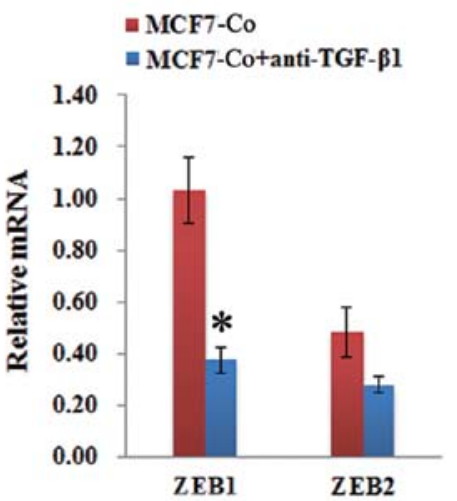

B

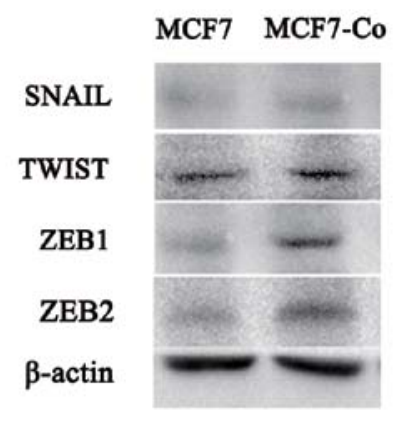

E

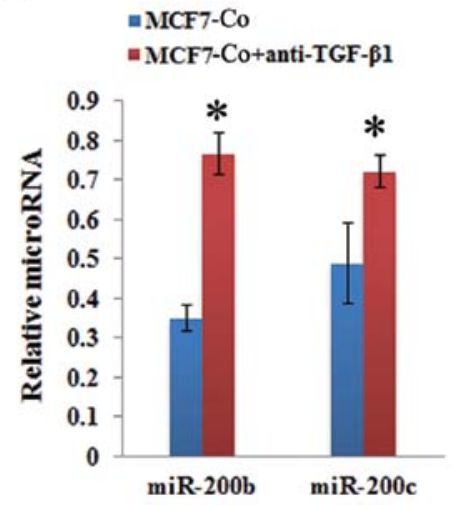

Figure 5. Paracrine TGF- $\beta 1$ targets ZEB/miR-200 regulatory loop. (A) Changes of the EMT-related transcriptional factors in mRNA levels in MCF7 cells following co-culture with hAD-MSCs for $72 \mathrm{~h}$. (B) Western blotting was applied to analyze the protein levels of these transcriptional factors. ("p $<0.05$ ). (C) Reduction of miR-200b and miR-200c levels was evident in MCF7-Co cells in comparison with those in MCF7. (D,E) Changes of ZEB1/2 and miR-200 in MCF7-Co cells after adding $8 \mu \mathrm{g} / \mathrm{ml}$ anti-TGF- $\beta 1$ antibody 3 days later. ${ }^{*} \mathrm{p}<0.05$.

Autocrine TGF- $\beta$ signaling is induced and required for the maintenance of a mesenchymal state in MCF7 cells. It has been well documented in studies with other EMT cell culture models that TGF- $\beta$ can cooperate with other signaling pathways to establish autocrine TGF- $\beta$ signaling. We hypothesized that prolonged co-culturing with hAD-MSCs might initiate an autocrine TGF- $\beta$ signaling in the MCF7 cells that would ultimately enforce its mesenchymal state. To test this, we first measured the expression of endogenous TGF- $\beta 1$, TGF- $\beta 2$, and TGF- $\beta 3$ mRNA levels in MCF7 cells at varying time points after co-culturing and we found that they were indeed progressively increased by co-culturing (Fig. 6A). We also observed that TGF- $\beta 1$ and TGF- $\beta 2$ proteins were being actively secreted by stable mesenchymal state MCF7-M cells (Fig. 6B).

To determine whether the response of cells to this endogenously synthesized TGF- $\beta$ is important for mesenchymal stability, we treated stable MCF7-M cells with an inhibitor of TGF- $\beta$ Receptor I, SB-505124. The addition of this inhibitor led to a time-dependent decrease in ZEB mRNA levels (Fig. 6C), which is consistent with the need for autocrine TGF- $\beta$ production by MCF7-M cells for endogenous ZEB transcription. Concomitant with the loss of $\mathrm{ZEB}$ expression, the expression of miR-200 was increased (Fig. 6D), and accompanied by hallmark epithelial features such as increased expression of E-cadherin and ZO-1 (Fig. 6E). These data collectively confirm that this epithelial reversion in MCF7-M cells was caused by the inhibition of the TGF- $\beta$ pathway.

\section{Discussion}

Previous studies investigating breast cancer cells that were directly co-cultured with MSCs have shown significant morphological alterations and the downregulation of E-cadherin protein expression in the breast cancer cells $(15,17)$. In this study, we found that hAD-MSCs can not only play a role in triggering EMT in MCF7 cells through paracrine TGF- $\beta 1$ signaling, but after prolonged co-culturing, they can also lead to the maintenance of MCF7 cells in a stable mesenchymal state. These results suggest that MSCs may promote breast cancer cell migration by stimulating and facilitating the EMT.

Due to the fact that $\mathrm{miR}-200 \mathrm{c}$ and $\mathrm{ZEB} 1$ regulate each other in a mutual, negative feedback loop (31), there is a critical threshold in the balance between miR-200 and ZEB levels that determines whether cells stably reside in a self-reinforcing epithelial or mesenchymal state $(34,35)$. Our findings have confirmed the previously held opinion that TGF- $\beta$ plays a pivotal role in this double feedback loop between miR-200c and ZEB1 to ultimately determine either an epithelial or mesenchymal phenotype (34). Paracrine TGF- $\beta 1$ secreted by hAD-MSCs results in an increased level of ZEB1 transcription in MCF7 cells that can reach a point where ZEB1 
A

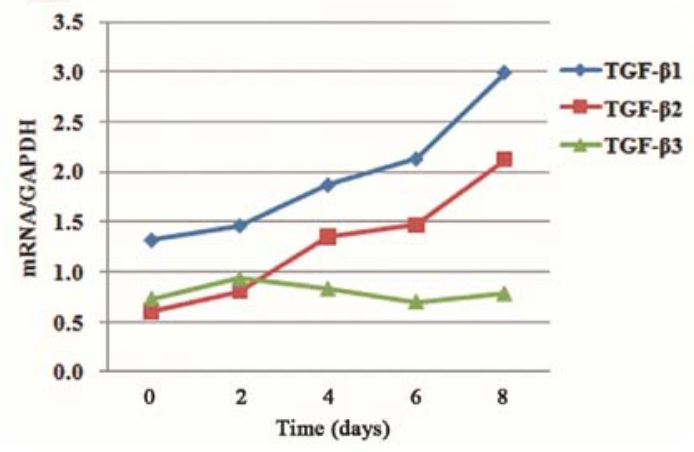

$\mathrm{C}$

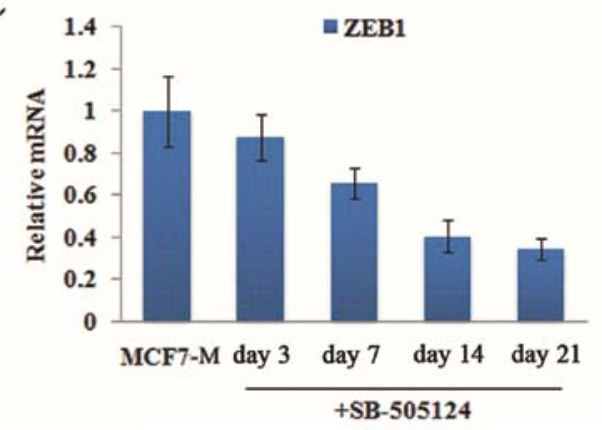

D

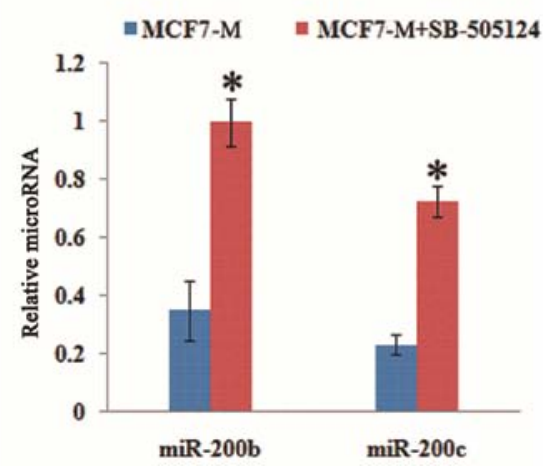

B
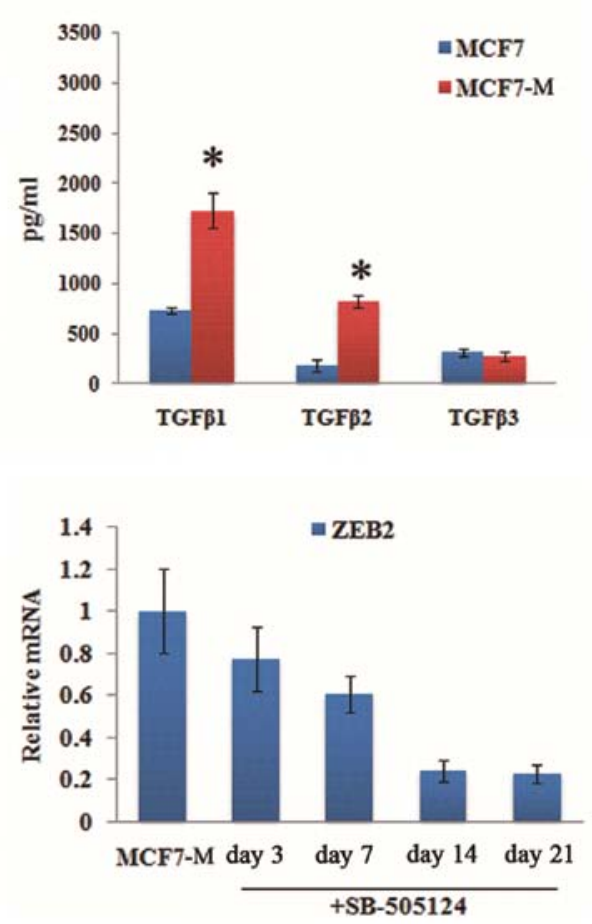

E

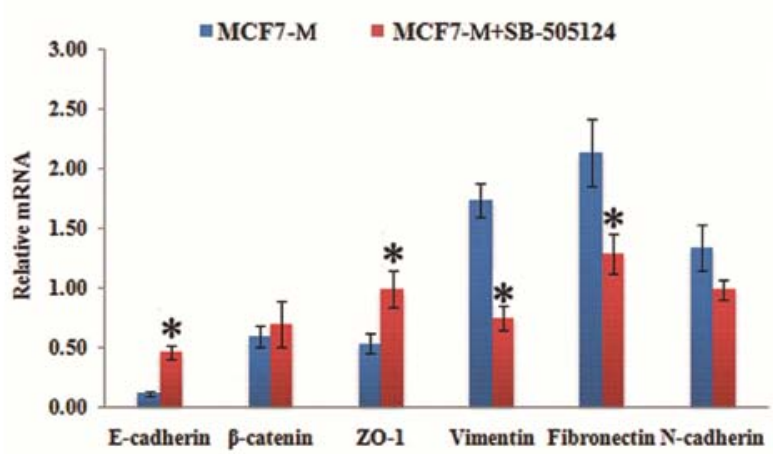

Figure 6. Autocrine TGF- $\beta$ signaling is initiated and required for the maintenance of a mesenchymal state. (A) Real-time PCR measurements of TGF- $\beta$ family members in MCF7-Co cells at different time points. (B) TGF- $\beta 1$, TGF- $\beta 2$ and TGF- $\beta 3$ secreted by MCF7 and MCF7-M cells into the culture medium were measured by ELISA. (C) Real-time PCR measurement of ZEBs in MCF7-M cells after inhibition of TGF- $\beta$ signaling using the TGF- $\beta$ RI inhibitor $(1 \mu \mathrm{M}$ SB-505124) at different time points. (D,E) Changes of miR-200 levels and accompanied EMT marker expression in MCF7-M cells following inhibition of TGF- $\beta$ signaling using the TGF- $\beta$ RI inhibitor (1 $\mu$ M SB-505124) for 14 days. ${ }^{*} \mathrm{p}<0.05$.

transcription can overcome the repression caused by miR-200. As ZEB1 proteins begin to accumulate, they can then repress the miR-200 family members, resulting in the progression of the EMT. The downregulation of paracrine TGF- $\beta 1$ signaling can reduce ZEB1 and ZEB2 expression, upregulate miR-200b and miR-200c, and inhibit the progression of the EMT. These results suggest that MSCs in the primary tumor microenvironment may play a pivotal role in triggering EMT through paracrine TGF- $\beta$ signaling, which is followed by the targeting of the $\mathrm{ZEB} / \mathrm{miR}-200$ regulatory loop in cancer cells. Our study provides a mechanistic explanation for how MSCs might facilitate cancer progression and metastasis in the tumor microenvironment.

Our report shows that co-cultured MCF7 cells display EMT changes in a time-dependent manner and that the transi- tion towards the mesenchymal state is only stabilized after 8 days of co-culturing. We also found that autocrine TGF- $\beta$ signaling is initiated and required for the maintenance of a stable mesenchymal state in MCF7 cells. Taken together, these results indicate that threshold changes in ZEB1/2 and miR-200 levels may potentially influence the autocrine TGF- $\beta$ signaling, which is important in determining the final outcome of the phenotypic state of the cell. It has been shown that TGF- $\beta 2$ is directly targeted by miR-141/200a in breast and colon cancer cell lines (28), which suggests that the loss of miR-200 family members during EMT might enhance autocrine TGF- $\beta$ signaling since the repression of TGF- $\beta 2$ is also alleviated. The subsequently initiated autocrine TGF- $\beta$ signaling could drive and sustain ZEB expression, which is necessary for stably maintaining the cells in a self-reinforcing mesenchymal state. 


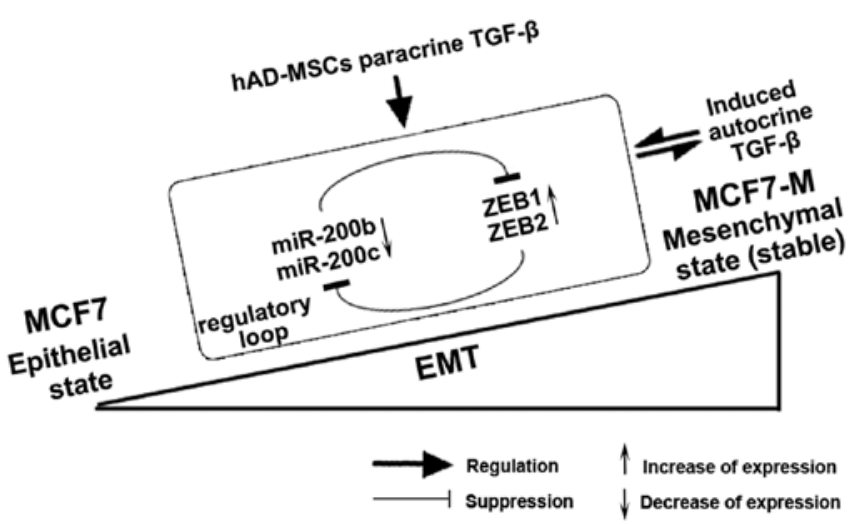

Figure 7. A proposed model of regulation of EMT in MCF7 cells by hAD-MSCs. We propose that hAD-MSCs regulate the establishment and maintenance of epithelial-mesenchymal transition in MCF7 human breast cancer cells. hAD-MSCs play a pivotal role in triggering EMT in MCF7 cells through paracrine TGF- $\beta$ signaling, which is followed by the targeting of the $\mathrm{ZEB} / \mathrm{miR}-200$ regulatory loop in cancer cells. This double-negative feedback loop between miR-200 family members and ZEB1 allows for the plasticity that exists between the cell's epithelial and mesenchymal states. Threshold changes in ZEB1/2 and miR-200 levels may potentially influence the autocrine TGF- $\beta$ signaling, which in turn could drive and sustain ZEB expression and is necessary for stably maintaining the cells in a selfreinforcing mesenchymal state.

Thus, we deduce that the paracrine TGF- $\beta$ signaling by MSCs during the long-term co-culture activates the autocrine TGF- $\beta$ signaling in MCF7 cells that is responsible for maintaining the MCF7 cells in a stable mesenchymal state (Fig. 7).

Due to the clinical importance of EMT-induced processes in understanding cancer progression, the inhibition of EMT is an attractive therapeutic approach that could have significant effects on disease outcome. Although the initial event triggering these changes is still poorly understood, our study suggests that targeting abnormal tumor-promoting paracrine signaling between the epithelial tumor and MSCs may be a promising approach for cancer prevention and treatment.

\section{Acknowledgements}

The authors thank Professor Zengxuan Song for the helpful discussion and critical reading of the manuscript, Yi Lin for the critical reading of the manuscript, Lianming Liao for the helpful discussion, and Kanghua Li and Jiansuo Zhou for their technical assistance. This project was supported by grants from the ' 863 Projects' of the Ministry of Science and Technology of PR China (no. 2011AA020100), the National Natural Science Foundation of China (no. 30830052 and 30911130363), the National Key Scientific Program of China (no. 2011CB964900), and the Program for Changjiang Scholars and Innovative Research Team in University-PCSIRT (no. IRT0909).

\section{References}

1. Gupta GP and Massague J: Cancer metastasis: building a framework. Cell 127: 679-695, 2006.

2. Weigelt B, Peterse JL and van't Veer LJ: Breast cancer metastasis: markers and models. Nat Rev Cancer 5: 591-602, 2005.

3. Guarino M, Rubino B and Ballabio G: The role of epithelialmesenchymal transition in cancer pathology. Pathology 39: 305-318, 2007.
4. Kang Y and Massague J: Epithelial-mesenchymal transitions: twist in development and metastasis. Cell 118: 277-279, 2004

5. Trimboli AJ, Fukino K, de Bruin A, et al: Direct evidence for epithelial-mesenchymal transitions in breast cancer. Cancer Res 68: 937-945, 2008.

6. Baum B, Settleman J and Quinlan MP: Transitions between epithelial and mesenchymal states in development and disease. Semin Cell Dev Biol 19: 294-308, 2008.

7. Hugo H, Ackland ML, Blick T, et al: Epithelial-mesenchymal and mesenchymal - epithelial transitions in carcinoma progression. J Cell Physiol 213: 374-383, 2007.

8. Thiery JP and Sleeman JP: Complex networks orchestrate epithelial-mesenchymal transitions. Nat Rev Mol Cell Biol 7: 131-142, 2006.

9. Yang J and Weinberg RA: Epithelial-mesenchymal transition: at the crossroads of development and tumor metastasis. Dev Cell 14: 818-829, 2008.

10. Brabletz T, Jung A, Spaderna S, Hlubek F and Kirchner T: Opinion: migrating cancer stem cells - an integrated concept of malignant tumour progression. Nat Rev Cancer 5: 744-749, 2005.

11. Mani SA, Guo W, Liao MJ, et al: The epithelial-mesenchymal transition generates cells with properties of stem cells. Cell 133: 704-715, 2008.

12. Gupta PB, Chaffer CL and Weinberg RA: Cancer stem cells: mirage or reality? Nat Med 15: 1010-1012, 2009.

13. Polyak K and Weinberg RA: Transitions between epithelial and mesenchymal states: acquisition of malignant and stem cell traits. Nat Rev Cancer 9: 265-273, 2009.

14. Hu M and Polyak K: Molecular characterisation of the tumour microenvironment in breast cancer. Eur J Cancer 44: 2760-2765, 2008.

15. Fierro FA, Sierralta WD, Epunan MJ and Minguell JJ: Marrowderived mesenchymal stem cells: role in epithelial tumor cell determination. Clin Exp Metastasis 21: 313-319, 2004.

16. Hombauer $\mathrm{H}$ and Minguell JJ: Selective interactions between epithelial tumour cells and bone marrow mesenchymal stem cells. Br J Cancer 82: 1290-1296, 2000.

17. Sasser AK, Mundy BL, Smith KM, et al: Human bone marrow stromal cells enhance breast cancer cell growth rates in a cell line-dependent manner when evaluated in 3D tumor environments. Cancer Lett 254: 255-264, 2007.

18. Chen J, Zhang ZG, Li Y, et al: Intravenous administration of human bone marrow stromal cells induces angiogenesis in the ischemic boundary zone after stroke in rats. Circ Res 92: 692-699, 2003.

19. Kumar S, Chanda D and Ponnazhagan S: Therapeutic potential of genetically modified mesenchymal stem cells. Gene Ther 15 : 711-715, 2008.

20. Karnoub AE, Dash AB, Vo AP, et al: Mesenchymal stem cells within tumour stroma promote breast cancer metastasis. Nature 449: 557-563, 2007.

21. Dwyer RM, Potter-Beirne SM, Harrington KA, et al: Monocyte chemotactic protein-1 secreted by primary breast tumors stimulates migration of mesenchymal stem cells. Clin Cancer Res 13: 5020-5027, 2007

22. Molloy AP, Martin FT, Dwyer RM, et al: Mesenchymal stem cell secretion of chemokines during differentiation into osteoblasts, and their potential role in mediating interactions with breast cancer cells. Int J Cancer 124: 326-332, 2009.

23. Zeisberg M and Kalluri R: The role of epithelial-to-mesenchymal transition in renal fibrosis. J Mol Med 82: 175-181, 2004.

24. Pardali K and Moustakas A: Actions of TGF-beta as tumor suppressor and pro-metastatic factor in human cancer. Biochim Biophys Acta 1775: 21-62, 2007.

25. Derynck R and Akhurst RJ: Differentiation plasticity regulated by TGF-beta family proteins in development and disease. Nat Cell Biol 9: 1000-1004, 2007.

26. Thiery JP, Acloque H, Huang RY and Nieto MA: Epithelialmesenchymal transitions in development and disease. Cell 139: 871-890, 2009.

27. Peinado H, Olmeda D and Cano A: Snail, Zeb and bHLH factors in tumour progression: an alliance against the epithelial phenotype? Nat Rev Cancer 7: 415-428, 2007.

28. Burk U, Schubert J, Wellner U, et al: A reciprocal repression between ZEB1 and members of the miR-200 family promotes EMT and invasion in cancer cells. EMBO Rep 9: 582-589, 2008.

29. Gregory PA, Bert AG, Paterson EL, et al: The miR-200 family and miR-205 regulate epithelial to mesenchymal transition by targeting ZEB1 and SIP1. Nat Cell Biol 10: 593-601, 2008. 
30. Park SM, Gaur AB, Lengyel E and Peter ME: The miR-200 family determines the epithelial phenotype of cancer cells by targeting the E-cadherin repressors ZEB1 and ZEB2. Genes Dev 22: 894-907, 2008

31. Bracken CP, Gregory PA, Kolesnikoff N, et al: A double-negative feedback loop between ZEB1-SIP1 and the microRNA-200 family regulates epithelial-mesenchymal transition. Cancer Res 68: 7846-7854, 2008.

32. Gregory PA, Bracken CP, Bert AG and Goodall GJ: MicroRNAs as regulators of epithelial-mesenchymal transition. Cell Cycle 7 : 3112-3118, 2008.
33. Cao Y, Sun Z, Liao L, Meng Y, Han Q and Zhao RC: Human adipose tissue-derived stem cells differentiate into endothelial cells in vitro and improve postnatal neovascularization in vivo. Biochem Biophys Res Commun 332: 370-379, 2005.

34. Gregory PA, Bracken CP, Smith E, et al: An autocrine TGF-beta/ ZEB/miR-200 signaling network regulates establishment and maintenance of epithelial-mesenchymal transition. Mol Biol Cell 22: 1686-1698, 2011.

35. Brabletz S and Brabletz T: The ZEB/miR-200 feedback loop - a motor of cellular plasticity in development and cancer? EMBO Rep 11: 670-677, 2010. 